\title{
Modelo de administración de proyectos de electrificación rural en Nicaragua incluyendo la generación en sistemas aislados
}

\author{
N. Blanco*, O. Salazar y E. Salazar \\ Facultad de Electrotecnia y computación, Universidad Nacional de Ingeniería (UNI) \\ PO Box 5595, Managua, Nicaragua \\ e-mail: blanconapoleon@yahoo.com
}

(recibido/received: 12-May-2009; aceptado/accepted: 12-Agosto-2009)

\begin{abstract}
RESUMEN
Contribuir al desarrollo de los sistemas aislados de generación de energía eléctrica fue el objetivo de la investigación descrita en este artículo. En este estudio se planteó una propuesta de modelo de administración de proyectos de electrificación rural, con el propósito de obtener una mayor eficiencia de la operatividad administrativa de la empresa o proyecto. Los sistemas aislados, no conectados a la red eléctrica convencional, brindan abastecimiento de energía eléctrica a las comunidades de las zonas rurales de Nicaragua; éstos abarcan las zonas más extensas e históricamente olvidadas del país, pero que a su vez poseen grandes potencialidades en recursos naturales y productivos; tal es el caso de las Regiones Atlánticas Norte y Sur. Para la propuesta del modelo de administración de proyectos de electrificación rural se empleó una metodología de análisis de proyectos basada en un esquema matricial con instrumentos de recopilación cualitativa. Basados en los resultados de esta investigación, se recomienda que en los proyectos de sistemas térmicos (usando derivados de petróleo), la forma de organización que mejor se adecua a las necesidades administrativas es del tipo empresa privada, con un enfoque jurídico de sociedad de responsabilidad limitada. En los proyectos de sistemas hidroeléctricos se recomienda una forma de organización privada como una sociedad anónima. Finalmente, en los proyectos de sistemas fotovoltaicos se recomienda una forma de organización privada bajo una empresa cooperativa.
\end{abstract}

Palabras claves: modelo de administración de proyectos; organización jurídica-administrativa.

\begin{abstract}
The objective of the research described in this article is to contribute to the development of remote rural electrical systems. The study sets out a proposed model for rural electrical project management, with the objective of achieving improved efficiency in operational management within companies or projects. Remote systems which are not connected to the conventional electricity grid, supply electricity to communities in rural areas of Nicaragua. They cover large areas which have, until now, been largely forgotten but which nevertheless have great potential in both natural resources and production. Such is the case in the Northern and Southern Atlantic Regions of Nicaragua. The proposed model for rural electrical project management uses a methodology of project analysis based on outline matrices using instruments of qualitative compilation. Based on the results of this research it is recommended that within projects of thermal power systems ( using by-products of petroleum) the form of organization best suited to management needs are those of private companies, in particular the limited liability company. In projects of hydroelectric systems the recommendation is for a form of private organization such as a joint-stock corporation. Finally in projects of photovoltaic systems the recommendation is for a form of private organization such as a business cooperative.
\end{abstract}

Keywords: model of project management; legal and administrative organization.

${ }^{*}$ Autor para la correspondencia 


\section{INTRODUCCIÓN}

La electrificación de un país y la diversificación de su matriz energética son pasos necesarios que una nación debe emprender para su desarrollo. Una parte importante de la estructura matricial eléctrica, tanto por su impacto social como por su aporte al desarrollo productivo, son los llamados sistemas aislados, los cuales abastecen de energía eléctrica a las comunidades de las zonas rurales de un país. En el caso de Nicaragua, la capacidad instalada de las unidades generadoras de energía eléctrica de los sistemas aislados apenas representan el $1.3 \%$ de la generación total del país. Así mismo éstas abarcan las zonas más extensas e históricamente olvidadas, pero a su vez poseen grandes potencialidades en recursos naturales, productivos y aprovechables principalmente en las regiones del Atlántico Norte y Sur.

Con el propósito de contribuir al desarrollo de los sistemas aislados del país, que son de vital importancia para el progreso socioeconómico de estas zonas, esta investigación se enfocó en la formación de una propuesta de administración de proyectos de electrificación rural, con el objetivo de obtener una mayor eficiencia de la operatividad administrativa de la empresa y/o proyecto.

\section{METODOLOGÍA}

Inicialmente el proceso de estudio consistió en la recopilación sistemática del funcionamiento administrativo de proyectos desarrollados (ver figura 1), y que necesariamente estaban en operación, con el objetivo de conocer los tipos de sistemas aislados existentes, a fin de familiarizarse con la manera en que éstos se administran. Basados en lo anterior, se pudo diferenciar cuáles de las figuras y formas administrativas realmente han dado resultados $\mathrm{y}$ son viables en Nicaragua.

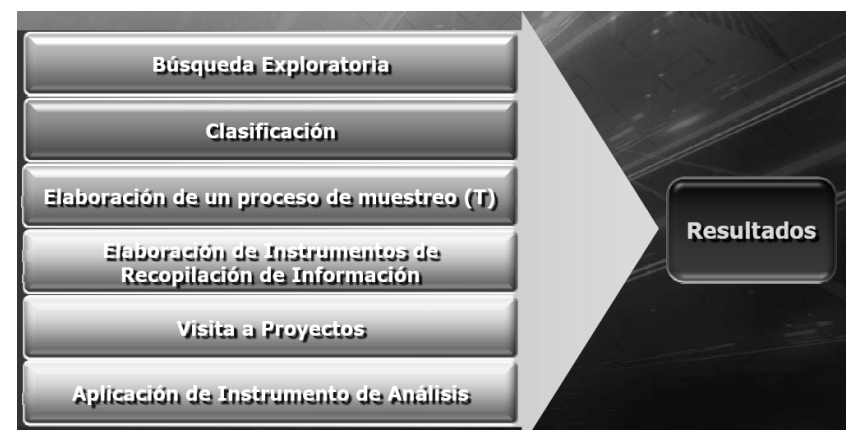

Fig 1. Esquema general del proceso metodológico de la investigación para elaborar modelo de administración de proyectos de electrificación en sistemas aislados.
Para lograr esto, se desarrolló una metodología de análisis basada en un esquema matricial con instrumentos de recopilación de información usando criterios técnicos, económicos, operacionales, de ejecución, financieros, sociales $y$ otros que contribuyeron a la interpretación de la eficiencia de las formas de organización administrativas (ver tabla .1)

\section{Tabla 1 Matriz de Proyectos de Electrificación} Rural

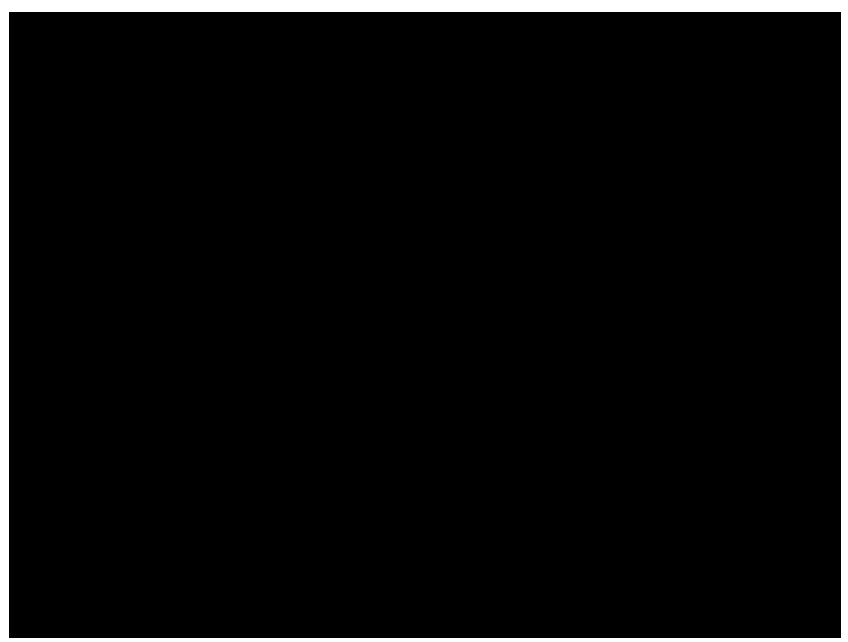

Este trabajo de investigación posee un enfoque más cualitativo que cuantitativo; por tal razón se hizo hincapié en los instrumentos de recolección de datos cualitativos. Se diseñaron instrumentos para la recolección de los datos como: entrevistas, cuestionarios $\mathrm{y}$ encuestas, los cuales fueron aplicados durante las visitas a los proyectos. Este proceso fue respaldado documentalmente durante su formulación. Se realizó la selección de la muestra de proyectos mediante un proceso estadístico; los datos recopilados en los proyectos permitieron realizar una interpretación sistemática para elaborar un modelo de administración que se ajuste a la realidad nicaragüense. Para el estudio se clasificaron los proyectos en: térmicos, hidroeléctricos y fotovoltaicos.

La estimación del tamaño de la muestra se realizó con la siguiente ecuación:

$\mathrm{n}^{\prime}=\mathrm{S}^{2} / \mathrm{V}^{2}$

$\mathrm{n}=\mathrm{n}^{\prime} / 1+\left(\mathrm{n}^{\prime} / \mathrm{N}\right)$

En donde $\mathrm{N}$ es la población (23 proyectos), $\mathrm{S}^{2}$ es la varianza de la muestra (normalmente se usa una muestra piloto o se toma este valor de otras experiencias relacionadas; en este caso se empleó un 12\%) 
expresada como la probabilidad de ocurrencia del fenómeno o de un evento esperado, el cual es establecido inicialmente. $\mathrm{V}^{2}$ es la varianza de la población al cuadrado y es equivalente al cuadrado del error estándar, y $\mathrm{n}^{\prime}$ representa el tamaño provisional de la muestra, la cual se corrige y se ajusta al tamaño de la población.

Una vez adquirida la información, el conocimiento y la experiencia de los proyectos valorados, el esfuerzo se concentró en estudiar, verificar, asimilar y comprender:

- Las teorías de administración más utilizadas en la operación de una empresa

- Los elementos de la administración como un proceso influyente en el buen éxito y desempeño del mismo como unidad empresarial.

- Los aspectos jurídicos de la empresa como formas de organización según las leyes permisibles de Nicaragua

- La síntesis condensada producto de la revisión de la ley de industria eléctrica en Nicaragua.

La información obtenida a partir de la experiencia en operación de proyectos de electrificación en sistemas aislados a nivel nacional, y el estudio de las formas organizativas para su operación $\mathrm{y}$, considerando los aspectos fundamentales de la administración no sólo desde la perspectiva operacional si no también desde la perspectiva jurídica nacional que rige los sistemas aislados de suministro de energía en zonas rurales; Permitió contar con suficientes elementos tanto teóricos como prácticos que posibilitaron establecer una propuesta de modelo de administración que se adecue a las características de los proyectos de sistemas aislados en Nicaragua, tomando como referencia las buenas prácticas aprendidas en los proyectos internacionales y sin dejar a un lado los conceptos y definiciones establecidos en la teoría de administración (ver figura 2).

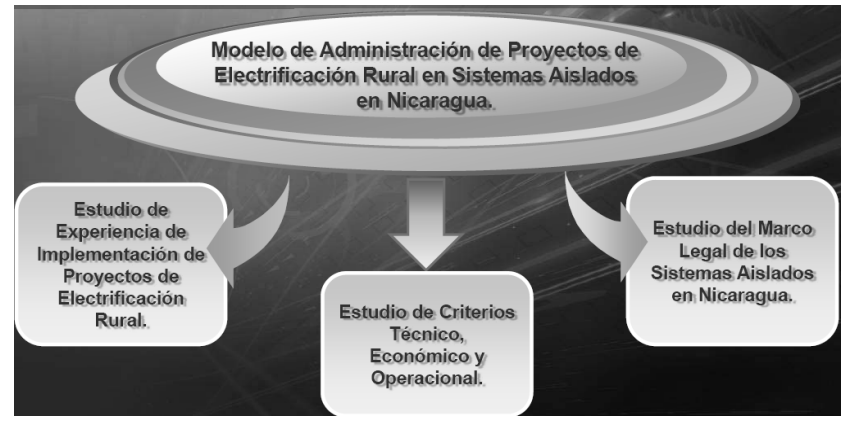

Fig 2. Elementos de base del modelo de propuesta de la administración de proyectos de electrificación en sistemas aislados.

\section{RESULTADOS Y DISCUSIÓN.}

En los proyectos térmicos los resultados relevantes fueron: los proyectos de plantas aisladas a base de combustible derivado de petróleo fueron ejecutados por la entidad estatal ENEL (Empresa Nicaragüense de Electricidad) quien opera bajo el sistema de agencia. En todos los proyectos se maneja una estructura tarifaria con un costo total mayor a $\mathrm{C} \$ 4 / \mathrm{kWh}$ (US $\$ 0.2 / \mathrm{kWh}$ ) aprobado por el INE (Instituto Nicaragüense de Energía); sin embargo se requiere de subsidio del Estado para la operación de las plantas, porque en la mayoría de los proyectos lo recolectado vía tarifa no cubre los costos de operación. Se maneja una sola estructura organizacional para el control y la dirección funcional y operacional de los sistemas de generación aislada, siendo la Dirección de Plantas Aisladas de ENEL la que encabeza este esquema de operación. La falta de sostenibilidad, comunicación ineficiente con la gerencia central y la falta de normativas en las actividades de mantenimiento tanto preventivo como correctivo, permiten concluir que la implementación y operación bajo el esquema de agencia de ENEL no es la más adecuada.

En los proyectos hidroeléctricos, el Ministerio de Energía y Minas (MEM) a través de la dirección de Pequeñas Centrales Hidroeléctricas (PCH) ha promovido, ejecutado y llevado a cabo un plan de seguimiento de los proyectos. En éstos existe un modelo de operación y administración conformado por un grupo comunitario, el cual es capacitado y supervisado por la $\mathrm{PCH}$ con una estructura definida y congruente entre sí, basado en un organigrama de trabajo y jurídicamente organizado como una sociedad anónima, cuyas transacciones económicas se realizan con apoyo del gobierno.

En los proyectos fotovoltaicos, la mayoría de proyectos de electrificación rural están enfocados en la generación de energía para sistemas de iluminación, por consiguiente los sistemas fotovoltaicos analizados poseen capacidades instaladas muy bajas, ya que estos sistemas no están dedicados a los sistemas productivos directamente. Los proyectos fotovoltaicos dependen mucho de la cooperación extranjera no sólo desde un punto de vista de financiamiento (la mayoría de los esquemas de financiamiento son con donación del 100 $\%$ de la inversión) e implementación, sino también desde un punto de vista técnico; esto se da por la capacidad que tienen estas entidades de brindar la tecnología necesaria para desarrollar los proyectos en el país, debido a que Nicaragua aún no posee la suficiente 
madurez tecnológica para hacer sustentables estos proyectos. Hay que destacar que la tarifa para los sistemas individuales tendría que ser muy alta para lograr pagar todos los equipos, por tal razón, para contribuir con los costos de inversión se recurre a mecanismos de participación comunitaria; ejemplo de ello es un proyecto ejecutado por el Grupo FENIX (Grupo voluntario del Programa de Fuentes Alternas de Energía de la Universidad Nacional de Ingeniería) en la comunidad de Sabana Grande en Somoto, donde un grupo de mujeres se organizaron con el nombre de Mujeres solares, con el objetivo de pagar parte de los equipos en forma de trabajo, denominando a este tipo de actividad "horas laborales".

Utilizando los resultados del análisis nacional, se determinó la propuesta del modelo (ver figura 3), con sus principales componentes, enfocados en proyectos con los tres tipos de energía conocidos: térmica, hidroeléctrica y fotovoltaica.

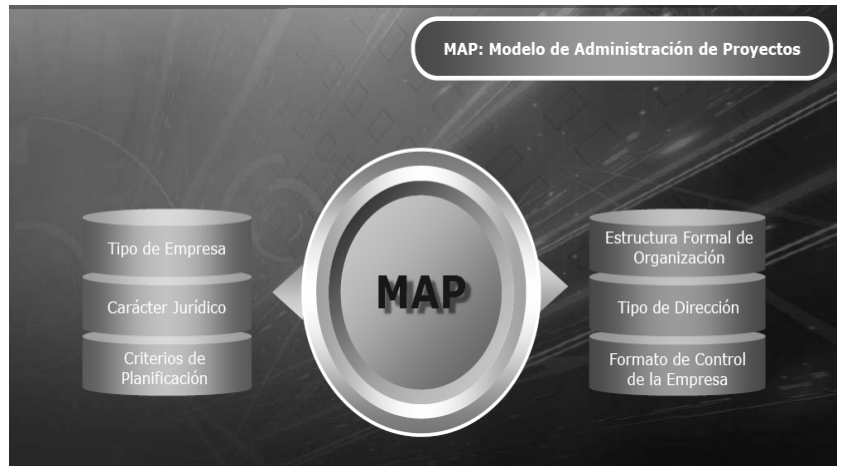

Fig 3. Esquema general de los componentes del modelo de administración de proyectos de electrificación en sistemas aislados.

Se propone la anulación total del modelo de Agencia como una forma de organización para operar el sistema que depende directamente de ENEL y se recomienda en primer lugar establecer una estrategia para sustituir paulatinamente este tipo de energía con un sistema a base de fuentes de energía renovable. Se propone en segunda instancia, el funcionamiento de sistemas eléctricos aislados a base de combustible fósil operados por pequeñas empresas de carácter privado que brinden el servicio de energía eléctrica. En relación a la personería jurídica de la empresa (ver figura 4, con los principales componentes del modelo), se plantea la constitución de una sociedad de responsabilidad limitada, cuyo capital estará dividido en participaciones sociales y se integrará por las aportaciones de cada uno de los socios, quienes no responderán de manera personal a posibles deudas y limitarán su responsabilidad a un valor establecido en el contrato social.

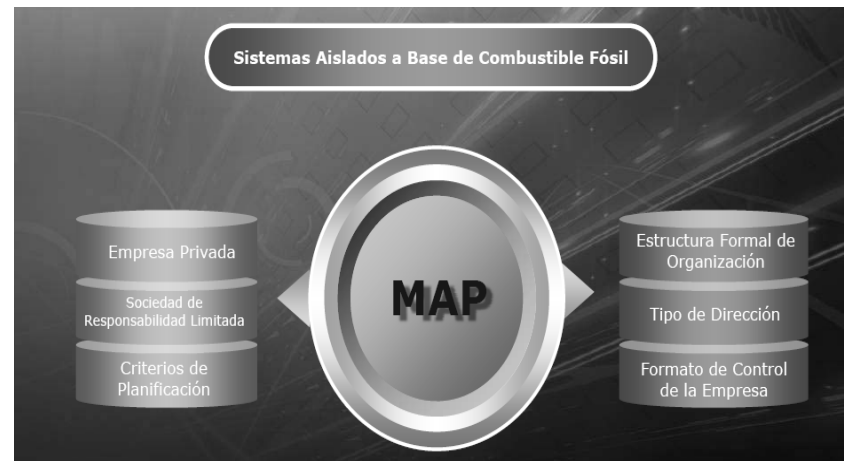

Fig 4. Esquema general de los componentes modelo de administración de proyectos de electrificación en sistemas aislados a base de combustible fósil.

La óptima operatividad de los sistemas hidroeléctricos se logrará por medio de empresas de carácter privado, que estarán directamente ligadas a la operación, bajo una concesión que regularice sus funciones. El Estado a través de sus principales instituciones como el MEM e INE regularán la operación y el servicio brindado de acuerdo al marco legal. La empresa privada tendrá una forma de organización mercantil definida (ver figura 5., con los principales componentes del modelo) como una sociedad anónima cuyo capital social se conformará por un fondo común dividido en acciones y cuyos miembros accionistas serán responsables hasta el monto de su respectivo capital.

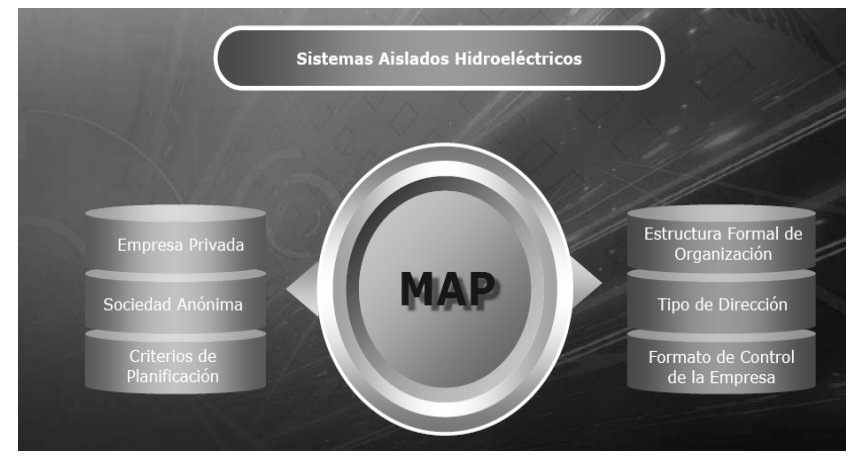

Fig 5. Esquema general de los componentes modelo de administración de proyectos hidroeléctricos (con pequeñas centrales hidroeléctricas como fuentes energéticas) en sistemas aislados.

En un sistema de generación aislada cuya fuente de energía sea fotovoltaica se plantea la formación de una pequeña empresa comunitaria (ver figura 6 , con los principales componentes del modelo) con un enfoque de servicio social y una visión de autosostenibilidad de carácter privado que administre el buen servicio de energía eléctrica y los activos fijos de la empresa para 
fines de mantenimiento de los equipos, con el propósito de satisfacer a los beneficiarios del proyecto. En relación a la personería jurídica de la empresa, se plantea la constitución de una sociedad cooperativa formada por un grupo indefinido de asociados con una responsabilidad social limitada al valor de sus aportes y una responsabilidad de la cooperativa para con terceros, al monto del patrimonio social.

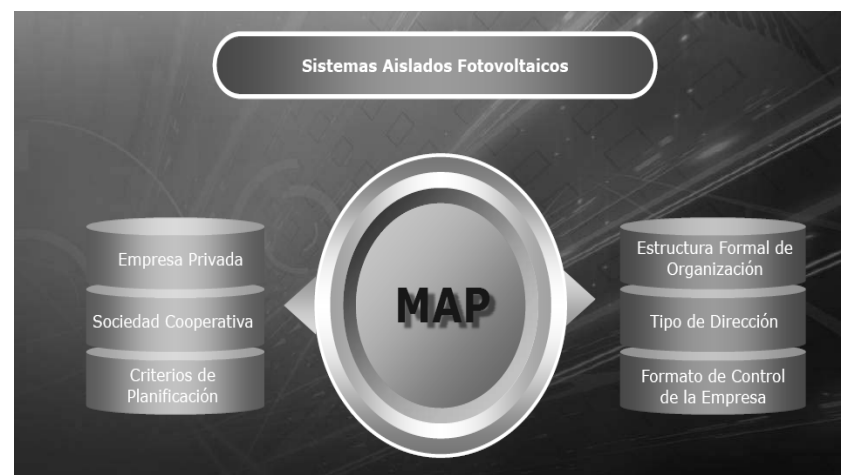

Fig 6. Esquema general de los componentes modelo de administración de proyectos fotovoltaicos de electrificación en sistemas aislados.

\section{CONCLUSIONES}

En conclusión, los proyectos de electrificación rural de los sistemas aislados poseen un vínculo directo con organismos extranjeros que los apoyan con inversión inicial, capacitación y organización comunal. Para alcanzar la sostenibilidad, los proyectos de electrificación rural tienen que ser independientes desde un punto de vista operativo y administrativo.

En los proyectos de sistemas térmicos la forma de organización que mejor se adecua a las necesidades administrativas es un tipo de empresa privada con un enfoque jurídico de sociedad de responsabilidad limitada. En los proyectos de sistemas hidroeléctricos se recomienda una forma de organización privada como una sociedad anónima. En los proyectos de sistemas fotovoltaicos se recomienda una forma de organización privada bajo una empresa cooperativa.

\section{REFERENCIAS}

Chiavenato, A. (2001), Administración Proceso Administrativo, I, Tercera Edición. Editorial Mc Graw Hill, México.ç

Chamoun, Y. (2002), Administración Profesional de Proyectos. La Guía. Primera edición. Mc Graw Hill, México.
Aguer, A., \& Pérez, E. (1997), Administración y Dirección de empresas, primera edición.

Código de Comercio de la República de Nicaragua y Compendio de Leyes Económicas y Mercantiles, (2007) Tomo I, Edición Revisada y Actualizada.

Muños, C. (1998), Como Elaborar y Asesorar una Investigación de Tesis, Primera Edición. Editorial Pearson, México.

Ángel, E. (2007), El Arte de Dirigir Proyectos, Segunda edición. Editorial alfaomega. México

Cuicar, O. (2002), Manual de cooperativas, Venezuela.

Orúe, J.(2008), Manual de derecho Mercantil, Segunda Edición. Editorial Hispamer, Nicaragua.

Hernández, R., Fernández, Carlos., \& Baptista, L. (2006), Metodología de la investigación, Cuarta Edición. Editorial Mc Graw Hill, México

Walpole, M. (1999) Probabilidad y Estadística para ingenieros, Sexta Edición. Editorial Prentice Hall, México.

Hodge B.J., W, P., L, M., \& Gales A. (1998), Teoría de la organización. Editorial Prentice Hall, México.

Cornell, W. (1980); Alexander Hamilton Organización de los negocios. Editorial alfa y Omega, México.

Ley General de Cooperativas de Nicaragua, Ley No. 499, 25 de enero 2005.

Pedro L. (1996), Administración y dirección de proyecto.

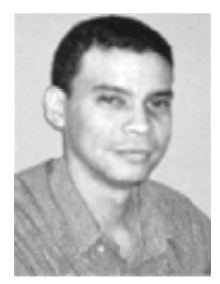

Napoleón Blanco se graduó de Ingeniero eléctrico, Máster en Gerencia de Proyectos de Desarrollo (UNI). Su área de investigación son los modelos económicos, factibles y viables para la implementación de sistemas de generación distribuida con fuentes de energía renovable como alternativa para el desarrollo del sector eléctrico de Nicaragua. 